\title{
Epigenetic regulation clocks the multigenerational olfactory imprinting in C. elegans
}

Madeleine Erard-Garcia ${ }^{1}$, Diana Andrea Fernandes de Abreu ${ }^{2}$, Antoine Gruet ${ }^{3}$, MariePierre Blanchard $^{4}$, Kevin Baranger ${ }^{5}$, Yoanne Clovis ${ }^{6}$, François Féron ${ }^{5}$ and Jean-Jacques Remy $^{2}$

${ }^{\mathbf{1}}$ Experimental and Molecular Immunology and Neurogenetics (INEM), Orleans University, CNRS UMR7355, Orléans, France

2 Institut Sophia Agrobiotech (ISA), CNRS UMR7254, INRAE 1355, Nice Côte d'Azur University (UCA), Sophia-Antipolis, France

${ }^{3}$ Human Oncology and Pathogenesis Program, Memorial Sloan Kettering Cancer Center, New York, NY, USA

4 Regional Imaging Platform, Montpellier Ressources Imagerie (MRI), BioCampus, Montpellier, France

5 Institut of Neurophysiopathology (INP), Aix Marseille University, CNRS UMR7051, Marseille, France

${ }^{6}$ Nemametrix Inc., Eugene, Oregon, USA

Correspondence to:

J.J. Remy, jean-jacques.remy@inrae.fr

Tel: +33492386418 


\begin{abstract}
Imprinting is an early sensory life experience that induces adult behaviours, such as mother recognition or homing. In a previous study, we demonstrated a striking olfactory imprinting in C. elegans that can be inherited over generations. When exposed to specific odorants during a timely controlled post-hatch period, C. elegans worms display during adulthood an enhanced migration towards these molecules. In order to unveil some of the genetic and epigenetic factors that are responsible for such a behavioural plasticity, we assessed the role of heterochronic genes using a candidate gene approach. We report here that translation of the Hunchback-Like 1 (HBL1) transcription factor in the sensory processing interneuron AIY, is a determining factor for olfactory plasticity timing in C.elegans. HBL1 may associate to the SPR1/CoREST co-repressor, the lysine demethylase SPR5/LSD1 and the histone deacetylase HDA3 to lengthen the plasticity period, whereas the translation initiation factor IFE-4 and the histone deacetylase HDA2 abridge it. We also observed that lengthened plasticity periods allow proportionally faster stable behavioral adaptation of $C$. elegans populations. We conclude that plasticity timing is a key factor, not only to transiently adapt individuals but also to stably adapt animal populations via multigenerational accumulation of experience.
\end{abstract}




\section{Introduction}

Behavioural adaptation is determined by a continuous intertwining of genetic and experiential factors, in particular during critical periods of plasticity. During these early life time-windows, juvenile animals receive and integrate environmental information that will imprint their adult behaviour.

Behaviour and cognitive abilities in birds are the best-described examples of how critical periods of plasticity are linked to behavioural adaptation (1). Bird species are classified along a spectrum spanning from precocial to altricial, with established differences in brain size at hatching, timing of brain development and behavioural complexity. Precocial birds, as Megapodes and Gallinaceous, rapidly express adult innate behaviours and become fully autonomous within hours or days post-hatching. In contrast, adult behaviours are expressed more slowly in altricial birds (parrots, passeriforms and song birds) that need up to weeks-long critical periods of nurturing, from environmental stimuli and parental care, to fully express adult behaviours. Evolution within the precocial/altricial spectrum is not limited to the bird class: growing complexity of cognitive capacities has also been linked to lengthening juvenile periods of plasticity, during primate (2) and hominid (3) evolution.

The C. elegans animal model is innately attracted to a number of olfactory cues, such as benzaldehyde (BA) and citronellol (CI) (4). These odorants bind the olfactory neuron receptors AWC (4) and the olfactory information is further processed by post-synaptic interneurons AIY. We show here that chemo-attraction is significantly reduced when induced during the course of the juvenile period, in comparison with the later stages of development. C.elegans worms however exhibit a critical period for chemosensory behaviour plasticity, which takes place during the first 12 hours after hatching $(5,6)$. 
We previously demonstrated that an exposition of juvenile animals to BA or CI during this critical period of plasticity induces a life-long behavioural adaptation via olfactory imprinting. At the adult stage, odour-exposed animals display an enhanced migration towards BA or CI (chemo-attraction) when compared to naïve animals (5). Strikingly, we also observed that olfactory imprinting is inherited either transiently or stably, depending on the number of consecutive worm generations exposed to BA or to CI during the period of olfactory plasticity. In the wild-type N2 strain of C.elegans, stable trans-generational inheritance of olfactory imprinting is precisely observed after five consecutive generations were odour-exposed (7).

The chemosensory behaviour is thus timely regulated in C. elegans worms. Since no behavioural timer genes have been identified in the worm so far, we used a candidate gene approach.

A well-described developmental heterochronic pathway controls the succession of the four L1 to L4 larval stages preceding the development of adult egg-laying worms. Mutations in the developmental heterochronic genes can disrupt the right succession of developmental stages by producing premature or delayed terminal differentiation phenotypes (8-10). We first considered the zinc finger transcription factor Hunchback-like-1 (HBL-1) as a chemoattraction timer, as HBL-1 controls the synchrony of several developmental events in $C$. elegans (11-15). In addition to be part of the developmental heterochonic pathway, HBL-1 is also the worm ortholog of the Drosophila melanogaster Hunchback transcription factor $(h b)$, a well-known timer of neuronal maturation in the fly (16). d4EHP, an eIF4E-like cap-binding protein, was shown to repress $h b$ while establishing axis polarity in the early drosophila embryo (17). Strikingly, one of the worm eIF4E translation initiation factor, IFE-4, was also reported to affect the translational efficiency of $h b l-1$ mRNA in C. elegans (18). 
When compared to the wild-type N2 worms, the juvenile hbl-1 mutants worms precociously displayed chemo-attraction while they lost olfactory plasticity and were unable to imprint odours. By contrast, the developmental expression of chemo-attraction was strongly delayed in the ife-4 mutants worms, compared to N2. In addition, the ife-4 worms keep a life-long ability to imprint odours, up to five times longer than wild-type animals. Moreover, the ife-4 mutation maintained high levels of the HBL-1 protein all over the development, suggesting the timing of olfactory plasticity and imprinting results from a translational regulation of $h b l-1$ by the translation initiator IFE-4.

We further reasoned that mutations in genes that control the timing of neuronal maturation would also affect the whole temporal settings of chemo-attractive responses. Neuronal timers in other species, as $h b$ and Tramtrack88 (Ttk88) in Drosophila $(16,19)$ and REST/NRSF in mammals $(20,21)$, are known to epigenetically control neuronal gene expression through association with the CoREST co-repressor and with conserved chromatin modifying factors as the Lysine-specific demethylase-1 (LSD-1) and the Class I Histone deacetylases (HDAC) (22). We report here that genetic inactivation of SPR-1, the worm ortholog of CoREST (23), or of the chromatin modifying factors SPR-5, HDA-2 and HDA-3, respectively the worm orthologs of the mammalian LSD1, HDAC-2 and HDAC-1, desynchronized the worm chemosensory behaviour.

We found that all mutations that extended the imprinting period in individuals proportionally decreased the number of generations to be imprinted before a stable change in chemosensory responses in populations. From these observations, we inferred that a stable behavioural change might be triggered after a fixed amount of heritable olfactory experience has been cumulated through generations. 


\section{Results}

\section{Biphasic implementation of chemo-attraction behaviour throughout C. elegans development}

C. elegans is innately attracted to BA and CI, with maximal attraction to the $1 / 100$ dilution. The life cycle of $C$. elegans is comprised of the in ovo embryonic stage, four larval stages and adulthood starts at 60 hours post-hatching.

We evaluated innate BA chemo-attraction at different time-points of N2 wild-type $C$. elegans development in order to assess its implementation (for a detailed description see Materials and Methods). BA chemo-attraction implemented throughout development (Figure 1). From 10 hours post-hatching, chemo-attraction progressed with a slow rate of implementation (flat slope of 0.015) while it progressed with a higher rate of implementation from 38 hours post-hatch to adulthood (steeper slope of 0.07), reaching its maximum level at the age of 96 hours. The same biphasic progression was observed for the implementation of chemo-attraction to CI (data not shown), sensed by the same AWC olfactory neuron (24). This biphasic implementation contrasts with the almost linear size progression of the $C$. elegans larvae (25).

Hunchback-like-1 (HBL-1) may act as a timer of chemo-attraction implementation during the course of development

To identify potential molecular timers, the early innate BA chemo-attraction implementation was assessed in larvae with mutations in $h b l-1$, in ife-4, and in worms with a transgene overexpressing hbl-1 solely in AIY. The 10, 15 and 26 hours old hbl-1 (ve18) and hbl-1 (mg285) mutant larvae migrate in BA gradients significantly faster than N2 wild-type larvae of same age (Figure 2A). 10 hours old hbl-1 larvae migrate as fast as 26 hours old N2 larvae, suggesting the absence of a functional $h b l-1$ gene hastened the onset of BA chemo-attraction by at least 16 hours. In contrast, the innate chemo-attraction to BA was significantly delayed 
in ife-4 (ok320) mutants and in ttx3::hbl-1 transgenic larvae, compared to N2. 26 hours old ife-4 and ttx3::hbl-1 larvae behaved as 10 hours old N2 larvae, indicating a mean delay of 16 hours.

We further compared the innate BA chemo-attraction in hbl-1, ife-4 and N2 worms during the whole course of development until the adult stage (Figure 2B). At early stages, BA attraction progressed two times faster in $h b l-1$ mutants than in N2, with progression slopes of 0.031 and 0.015 , respectively. Impaired motility at later stages in $h b l-1$ mutants is due to welldescribed morphological defects (12). The mean migration of ife-4 (ok320) mutants in BA chemo-attraction tests becomes similar to N2 after 26 hours post-hatch, implementing with the same rate (slope of 0.07).

These observations prompted us to investigate $h b l-1$ and ife- 4 transcription levels in the AIY interneuron of wild-type N2 using fluorescent reporters (Supplemental Figure 1 A and B). To assess $h b l-1$ expression, we analyzed a GFP reporter construct containing the $h b l-1$ promoter and $1 \mathrm{~kb}$ of the $h b l-1$ gene (reporter strain BW1932 (12)). To examine the pattern of ife-4 expression we used the transgenic extra-chromosomal array ife-4::GFP(sEx15051). In addition, a reporter construct expressing CherryFP under the AIY specific $t$ tx-3 promoter B was used to label the AIY interneuron (strain ott4607; kind gift from O. Hobert). We observed the expression of the $h b l-1:: g f p-h b l-1$ reporter overlaps with the AIY specific label at all stages (Supplemental Figure 1A), suggesting hbl-1 transcription in AIY might not be down-regulated after the early olfactory plasticity and imprinting period. Interestingly, ife4::GFP is detected in AIY but not before 12 hours post-hatch, which corresponds to the end of the imprinting period. The ife-4::GFP reporter is also expressed in other neuronal cell bodies (Supplemental Figure 1B).

We therefore hypothesized that IFE-4 protein could inhibit HBL-1 protein synthesis ending the period of plasticity. In order to test this, we quantified the levels of HBL-1 in ife-4, in hbl- 
1 mutants and in wild-type N2 worms at several developmental stages, using home made HBL-1 specific antibodies (Supplemental Figure 2). In N2, the level of HBL-1 in AIY might be higher at the earlier than at the later larval stages. HBL-1 in N2 larvae was however only detected after loading high amount of larval protein extracts (Supplementary Material and Methods). This technical limitation probably explains why a down-regulation of HBL-1 is not observed in N2.

The ife-4 (ok320) mutants synthesised higher levels of HBL-1 protein than N2 at all developmental stages, indicating IFE-4 is indeed acting as a translational inhibitor of $h b l-1$ (Supplemental Figure 2).

Compared to wild-type, chemo-attraction is precociously expressed in $h b l-1$ mutants, while over-expression and over-time persistence of HBL-1 in the ife-4 mutants and in the ttx3::hbl-1 transgenic animals delayed chemo-attraction expression. Taken together this body of data strongly suggests that $h b l-1$ translation in the AIY neuron is a main timing factor for chemo-attraction expression during early development.

\section{Plasticity time-length is negatively correlated to chemo-attraction expression}

We hypothesised the expression of innate chemo-attraction might be put on hold during the olfactory plasticity period during which olfactory experience can be imprinted. If true, delayed expression of chemo-attraction would be linked to lengthened imprinting periods, while precocious expression of chemo-attraction would be linked to shortened imprinting periods.

In order to test this hypothesis, precocious $h b l-1$ and delayed ife-4 and ttx-3::hbl-1 mutants were exposed to BA 1/300 during 24 hours post-hatch, a time interval that spans the wild-type imprinting period (Figure 3). The behaviour of BA-exposed and the behaviour of unexposed worms from each strain were then compared at four days post-hatch. Imprinting 
index is calculated by subtracting the responses of naive from the responses of odour-exposed worms (for a detailed description see Materials and Methods). A positive imprinting index thus indicates odour-exposed worms migrated faster than naïve-unexposed worms.

BA pre-exposure enhanced adult BA attraction in N2 wild-type worms, with a positive mean imprinting index of $2.1 \pm 0.25$. BA pre-exposure also enhanced adult BA chemoattraction in the delayed ife-4 and ttx-3::hbl-1 mutant worms with positive imprinting indices of $1 \pm 0.25$ and $1.7 \pm 0.3$, respectively (Figure 3). Early BA exposure of the precocious $h b l-1$ (ve18) and hbl-1 (mg285) mutants induced by contrast a decreased chemo-attraction to BA. This led to negative mean imprinting indices of $-0.8 \pm 0.3$ for hbl-1 (ve18) and of - $1.8 \pm 0.3$ for hbl-1 (mg285) worms, respectively (Figure 3).

\section{Histone-modifying factors are behavioural plasticity timers}

In order to identify other chemo-attraction timers, we considered the REST (RE1Silencing Transcription Factor) pathway. REST is a mammalian timer of neuronal differentiation (21). It mediates transcriptional repression in association with its co-repressors mSin3 and CoREST. In addition, the REST-CoREST complex can recruit histone modifiers as histone deacetylases (HDACs) and histone demethylase (LSD1) for long-term silencing of neuronal genes $(21,22)$. In the worm, no REST ortholog has been characterized, but a core complex consisting of SPR-1 (CoREST ortholog), SPR-5 (LSD1/KDM1 ortholog) and, possibly yet unknown, HDACs, might be recruited to the regulatory region of target genes through interaction with unidentified transcription factors with REST function (23).

HBL-1 may have a REST-like activity in the C. elegans neurons, as Hunchback in Drosophila melanogaster. HBL-1 may thus associate with CoREST/SPR-1, LSD1/SPR-5 and with some HDACs to time the worm chemosensory behavior. If so, mutations in the corresponding genes would also modify the onset of chemo-attraction. 
In order to test this hypothesis, the innate chemo-attraction of several mutants of this putative timing complex was assessed. Two spr-1 (ar205) or spr-1 (ar200) mutants, lacking the Cterminal SANT domain of SPR-1 (23), exhibited a precocious innate BA chemo-attraction at 26 hours post-hatch, similarly to hbl-1 mutants (Figure 4A). We assessed three spr-5 alleles ar197, by101 and by119. Only spr-5 (by119) larvae exhibited a precocious innate chemoattaction, compared to wild-type. This discrepancy may be due to the nature of the mutations. The structure of the putative worm SPR-1/SPR-5 complex is unknown. Based on the 3D structure of the mammalian CoREST/LSD-1 complex (26), the ar197 and by101 mutations may only affect the C-terminus of the SPR-5 protein, leaving intact the putative SPR-1 interacting domain. By contrast, the by119 allele, carrying a premature stop codon in the FAD-binding domain, may impair the formation of a SPR-1/SPR-5 complex.

The C. elegans Class I HDAC is made of three genes, hda-1, hda-2 and hda-3. hda-1 is needed for the development of the nervous system and axon guidance (27). The hda-1 mutants are sterile and severely uncoordinated, and were thus excluded from our screen. We thereafter compared the innate BA chemo-attraction timing of worms with deletions in hda-2 and $h d a-3$, the two remaining Class I HDAC genes, as well as in $h d a-4$, a Class II HDAC gene involved in chemoreceptor gene expression (28). While the hda-4 (ok518) deletion had no effect on chemo-attraction timing, the hda-2 (ok1479) and hda-3 (ok1991) deletions produced heterochronic phenotypes. The hda-2 (ok1479) larvae behaved as the delayed ife-4 (ok320) larvae, while the hda-3 (ok1991) larvae precociously expressed BA chemo-attraction compared with wild-type N2 (Figure 4A).

\section{Chemo-attraction timing is linked to the processing of olfactory experience}

The mutant worms we have assessed can be divided into three groups according to innate BA chemo-attraction phenotypes displayed at 26 hours (Figure 4A). A first group with wild-type BA chemo-attraction comprises spr-5 (ar197), spr-5 (by101) and hda-4 (ok518). A second 
group includes ife-4 (ok320), hda-2 (ok1479) and the ttx-3::hbl-1 transgenic worms that display a delayed innate BA chemo-attraction. A third group is made of the hbl-1 (mg285), hbl-1 (ve18), spr-1 (ar205), spr-1 (ar200), spr-5 (by119) and hda-3 (ok1991) mutants that display a precocious innate BA chemo-attraction. In order to test how chemo-attraction implementation affects the olfactory imprinting, mutants within the three groups were exposed to BA 1/300 during 12 hours post-hatch, the wild-type imprinting period (Figure 4B). In wild-type N2, BA-exposure enhances adult response to BA, compared to naïve N2 unexposed worms. All mutants belonging to the first and second groups imprinted BA as wild-type N2 worms. Strikingly, early BA exposure of mutants belonging to the third group decreased BA-chemo-attraction. Mean imprinting indices were found all negative for worms carrying the spr-1 (ar205), spr-1 (ar200), spr-5 (by119) and hda-3 (ok1991) mutations, which reflect a lower BA chemo-attraction for BA-experienced than for naïve worms. Worms with

these mutations do keep a lifelong negative imprint of early experience. As shown in Figure 4 inset, our behavioural screen thus disclosed a strict correlation $(\mathrm{R}=0.822)$ between the early onset of BA chemo-attraction (Mean Migration Index to BA 1/100 at 26 hours) and the way early BA experience is accounted by adults (Mean Imprinting Index).

\section{Delayed onset of chemo-attraction is associated with extended time-lengths of olfactory plasticity}

Mutations that delayed the expression of innate BA chemo-attraction might also extend the BA imprinting plasticity period. To test this, hda-4 (ok518), hda-3 (ok1991), hda-2 (ok1479), ife-4 (ok320) and ttx-3::hbl-1 mutants were BA-exposed during 0-12, 0-60, 12-36, or 36-60 hours post-hatch. As shown in Table 1, N2 and hda-4 (ok518) worms exclusively imprinted the BA-chemo-attraction when exposed during time periods encompassing the wild-type N2 critical period (time intervals 0 to 12 and 0 to 60 hours). Negative imprinting in 
hda-3 (ok1991) worms happened during the same time periods. In contrast, the competence for producing an imprint persisted far beyond the wild-type olfactory plasticity period in the hda-2 (ok1479), ife-4 (ok320) and ttx-3::hbl-1 mutant worms. hda-2 (ok1479) kept on generating BA imprints up to the age of 36 hours (as indicated by the 12-36 hours period), displaying an imprinting plasticity period at least three times longer than N2. ife-4 (ok320) mutants and $t t x-3:: h b l-1$ transgenics imprinted at all stages, displaying an imprinting plasticity period at least five times longer than N2. Noteworthy, the larval development in $h d a-2$ (ok1479), ife-4 (ok320) and in the ttx-3::hbl-1 mutants was unaffected (data not shown), suggesting these worms imprint odours efficiently at any age, irrespective of the developmental stage.

\section{Cumulated multi-generational experience determines the rate at which behaviour is} stably reshaped

Olfactory imprinting is always passed to the unexposed F1 generation, but it is not transmitted to the unexposed F2 generation in wild-type N2 worms (7). However, if five successive worm generations are odour-exposed, all the following unexposed generations $(\mathrm{F} 5+1,+2,+\mathrm{N}$ unexposed generations) inherit the imprint (7). The multi-generational olfactory-experience has stably reshaped the chemo-attractive behaviour in worm population.

In this paper, we have identified mutants with variable imprinting periods: 12 hours long for N2, hda-4 (ok518), 36 hours long for hda-2 (ok1479) and at least 60 hours long for ife-4 (ok320) and ttx-3::hbl-1. Considering this unique body of data, we could test if, as suggested (29), the accumulation of multigenerational imprinted experience is responsible for stably reshaping the behaviour of an animal population.

To test this hypothesis, we first determined if variable lengths of plasticity periods affected the rate at which BA-imprinting was stably assimilated by the progeny. Previous study showed that if imprinting is still present at the second, a fortiori at the fifth unexposed 
generation shown in Table 2, it will be permanently fixed and inherited by all following generations in worm populations (7). In order to assess this question, different mutants were BA-exposed for 60 hours post-hatch for five consecutive generations. Imprinted BAexposition was stably inherited by the N2 or hda-4 (ok518) after five generations (Ngen = 5) had been BA-exposed but not before (Table 2). Strikingly, in the case of mutants with lengthened plasticity periods, the stable assimilation of BA-imprinting needed less generations than for N2 to happen. For the hda-2 mutant, that has a 36 hours plasticity period, the stable assimilation of BA-imprinting happened after four generations of odour-exposure $(\mathrm{Ngen}=4)$. Moreover, for ife-4 (ok320) and ttx-3::hbl-1 mutants, that have a plasticity period of at least 60 hours, the assimilation of the BA-imprints happened immediately at the first generation $(\mathrm{Ngen}=1)$. Figure 5A summarizes how the imprinting plasticity period influences the number of generations required before imprinting assimilation operates. These results strongly suggest that a total of 60 hours of olfactory imprinting during the plasticity period will reshape the innate behaviour of worm populations. In the case of wild-type N2 worms, one generation of imprinted BA-exposition would produce 1/5 (around 12 hours) of the amount of imprints required for behavioural reshaping. In order to test this hypothesis, consecutive generations of ife-4 (ok320) mutants were BA-exposed either during 12, 36 or 60 hours post-hatch per generation, corresponding respectively to the N2, to the hda-2 (ok1479), and to the whole ife-4 (ok320) imprinting plasticity periods (Figure 5B). It took four generations with multigenerational 36 hours long exposures, and five generations with multigenerational 12 hours long exposures before stabilisation of the behaviour. As already shown, in ife-4 mutants able to imprint during at least 60 hours, a single exposure of 60 hours led to stable inheritance. We inferred that at least 60 hours of imprinted olfactory experience must be accumulated to reach the amount of olfactory experience required for a stable behavioural change. 


\section{Discussion:}

We pinpointed several molecular mechanisms that control the temporal settings of chemosensory adaptation in C. elegans individuals and populations. Our data suggest that temporal shifts in neuronal maturation of individuals by epigenetic mechanisms could dramatically influence how and how fast experience can be cumulated, accounted and integrated by animal populations.

Chemo-attraction implementation progresses throughout wild-type worms development, despite a latency period concomitant with the olfactory plasticity period. Worms lacking functional hbl-1, hda-3, spr-1 and spr-5 genes express chemo-attraction precociously without this latency period. Such precocity suppressed olfactory imprinting and modified the processing of early olfactory experience. This modified processing was previously observed in the imprinting mutants sra-11 and $t t x-3$ (5), but the mechanisms implicated in this phenomenon remain unclear. In contrast, worms in which hbl-1 was specifically overexpressed in AIY interneuron and worms in which hbl-1 expression was maintained overtime due to the lack of the putative inhibitor IFE-4 displayed a lengthened latency period of plasticity, up to adulthood. This could be explained by the maintenance of AIY in an immature highly plastic state by the continuous expression of the HBL-1 timer.

The timing of chemosensory adaptation we describe here displays some analogy with the timing of larval development in C. elegans $(10,11)$. Indeed, in both cases, loss of function or persistent expression of timers blocks the right progression of the temporal series. In the developmental heterochronic pathway, loss of function mutations in the lin-14, lin-28 or lin-41 genes result in skipping the L1, L2 or L3 larval stages, respectively, and promote premature expression of the next stage Instead, gain of function mutations of these genes 
produce overtime reiteration of the same respective stages. In the behavioural heterochrony pathway, loss of function in the hbl-1, spr-1, spr-5 and hda-3 genes induce a premature expression of the innate chemosensory responses, skipping the imprinting plasticity period, while HBL-1 gain of function in ife-4 and ttx3::hbl-1 mutants maintained the ability to imprint odours over life-time. These results seem to indicate that any behaviour open to experience-induced adaptation will not be expressed while behavioural adaptation can happen.

LSD1/HDAC1/2/CoREST (LHC) complexes modulate gene expression through interaction with different zinc-finger transcription factors (30), including REST/NRSF and Tramtrack88 in mammalian and fly neurons, respectively (19). Besides SPR-5/LSD1, SPR1/CoREST may bind members of the HDAC family through its two SANT domains (23). spr1 and hda-3 mutants display a precocious expression of the innate chemo-attraction, suggesting SPR-1 could associate with HDA-3 to control AIY plasticity timing.

Our results support the existence of a worm LHC complex comprised of SPR1/CoREST, SPR-5/LSD1 and HDA-3/HDAC1. In the absence of a REST-like protein it may associate with HBL-1 to regulate gene expression in C. elegans neurons. Interestingly in a study focused on the epigenetic control of olfactory receptor (OR) choice by mouse olfactory neurons (ORN), it has been shown that the role of LSD-1 is to remove the repressive methylation me2 and me3 marks on the lysine 9 of H3 histones (31). The down-regulation of LSD-1 as ORN mature timely links OR gene expression to neuronal differentiation in the mouse. Our results suggest that SPR-5/LSD-1 may also control OR genes expression in the worm; the absence of OR repression in spr-5 mutants would impairs chemo-sensory neuron plasticity.

Demethylation by LSD1 and deacetylation by HDAC result in chromatin compaction and prevent transcription factors, regulatory complexes and RNA polymerases to bind DNA, 
all well known epigenetic mechanisms. Interestingly, LSD1 is also an integral component of the Mi-2/nucleosome remodelling and deacetylase (NuRD) complex (32), which mediate transcription repression by distinct factors including Hunchback and Ikaros (33). Noteworthy, Ikaros, Hunchback and Tramtrack88 also recruit LSD1 (34).

We report here opposite roles for the C. elegans histone acetylases HDA-2 and HDA-3 in the regulation of the chemosensory behaviour adaptation. Based on primary sequences comparison (data not shown), the worm HDA-3 and HDA-2 could be the respective orthologs of the mammalian HDAC1 and HDAC2. Strikingly, HDAC2, but not HDAC1, negatively regulates learning and memory in the mouse. HDAC-2 acts as a repressor of synaptic remodelling and plasticity genes (35) and HDAC2 inhibition restores cognitive abilities in a mouse model of Alzheimer's disease (36). Our findings suggest the opposite functions for HDA-3/HDAC1 and HDA-2/HDAC2 in neuronal plasticity and maturation might be evolutionary conserved. A putative HBL-1/LHC complex would thus epigenetically control the timing of the plasticity periods within AIY interneurons. Furthermore, IFE-4 seems to be essential to inhibit HBL-1 translation.

Our results suggest that worms produce heritable olfactory imprints exclusively during the AIY plasticity period, while the innate chemo-attraction is repressed. In multigenerational imprinting situation, odour-exposed worms would add imprints they produce to imprints inherited from past generations, leading to imprint accumulation throughout generations, reaching an effective threshold that triggers integration and stable inheritance of the newly acquired experience.

In this paper, we tried to unveil some of the relationships between the different temporal variables that may determine the rate at which multigenerational experience stably change animal behaviours. As schematically outlined in Figure 6, it could be inferred from our data that 1) the onset of an adult behaviour is inhibited while the period of plasticity is 
maintained; 2) the period of plasticity delimits the amount of individual acquired experience imprinting the adult behaviour, and; 3) the amount of acquired experience constraints the adaptation of the populations. Specific genetic and epigenetic factors should time the functional maturation of neurons responsible for a given behaviour. Then the time-length of juvenile periods of behavioural plasticity would in turn determine the amount of experience that can be acquired during a generation. Stable changes in population behaviour would be acquired through the integration of cumulated heritable experience over multiple generations. The rate of stable behavioural adaptation would thus be determined by the amount of experience acquired by each generation. In brief, neuronal maturation speed is expected to be responsible for the rate innate behaviour change, according to ancestral experience.

Through yet unknown mechanisms, an environmentally induced reversible adaptation may evolve towards stable expression without triggering environmental stimuli (29). In particular, it is now well accepted that epigenetic alterations may contribute to establish phenotypic divergence among genetically identical animals exposed to different experiences (37). Other works also indicate that temporal shifts of gene expression during development have been recognized as potential drivers of speciation $(11,38,39)$. Evidence also indicates that learning and behavioural/cultural shifts could support fast reproductive isolation $(40,41)$. The present work suggests that temporal shifts in epigenetically controlled neuronal maturation could entail dramatic consequences on stable behavioural adaptations. 


\section{Material and Methods}

C. elegans strains were maintained at $20^{\circ} \mathrm{C}$ under standard growth conditions (Brenner, 1974). Synchronized populations of worms were used to perform all experiments. All confocal images were acquired using a Zeiss LSM780 microscope. The HBL-1 antibodies were custom-made. Supplementary Materials and Methods contain detailed descriptions on the worm strains, and on imaging, Western blotting and chemotaxis assay protocols. Chemotaxis assay is schematized in the Supplemental Figure 3.

We have no competing interests.

\section{Footnotes}

Author contributions MG carried out the molecular lab work, participated in data analysis, FF participated in the design of the study; DAFdA edited the manuscript; AG, KB and YC carried out molecular lab work and behavioural experiments; MPB collected microscopy data; JJR conceived and coordinated the study, performed experiments, carried out the statistical analyses and draft the manuscript. All authors gave final approval for publication.

\section{Acknowledgements}

We thank O. Hobert, D. Baillie, M. Delattre and I. Greenwald for worm strains and plasmids. Other strains used in this study were provided by the Caenorhabditis Genetics Center, which is funded by the National Institutes of Health (NIH) Office of Research Infrastructure Programs (P40 OD010440). This work was supported by a grant of the “Agence Nationale de la Recherche” ANR-12-Bioadapt-0022. 


\section{References}

1. Gill F.B. Ornithology. 3e ed. W.H. Freeman and Company, New York, 758 p. (2007).

2. Wobber V., Wrangham R., \& Hare B. Bonobos exhibit delayed development of social behaviour and cognition relative to chimpanzees. Curr.Biol. 20, 226-230 (2010).

3. Coqueniot H., Hubli J.J., Veillon F., Houet F. \& Jacob T. Early brain growth in Homo erectus and implications for cognitive ability. Nature 431, 299-302 (2004).

4. Bargmann C.I., Hartwieg E. \& Horvitz HR. Odorant-selective genes and neurons mediate olfaction in C. elegans. Cell 74, 515-527 (1993).

5. Remy J.J. \& Hobert O. An interneuronal chemoreceptor required for olfactory imprinting in C. elegans. Science 309, 787-790 (2005).

6. Jin X., Pokala N. \& Bargmann C.I. Distinct Circuits for the Formation and Retrieval of an Imprinted Olfactory Memory. Cell 164, 632-643 (2016).

7. Remy J.J. Stable inheritance of an acquired behaviour in Caenorhabditis elegans. Curr Biol 20, 877-878 (2010).

8. Ambros V. \& Horvitz H.R. Heterochronic mutants of the nematode Caenorhabditis elegans. Science 226, 409-416 (1984).

9. Wightman B., Ha I. \& Ruvkun G. Posttranscriptional regulation of the heterochronic gene lin-14 by lin-4 mediates temporal pattern formation in C. elegans. Cell 75, 855-862 (1993).

10. Moss E.G. Heterochronic genes and the nature of developmental time. Curr Biol 17, 425434 (2007).

11. Pasquinelli A.E. \& Ruvkun G. Control of developmental timing by microRNAs and their targets. Annu. Rev. Cell Dev. Biol. 18, 495-513 (2012).

12. Fay D.S., Stanley H.M., Han M. \& Wood W.B. A Caenorhabditis elegans homologue of hunchback is required for late stages of development but not early embryonic patterning. Dev Biol 205, 240-253 (1999).

13. Abrahante J.E. et al. The Caenorhabditis elegans hunchback-like gene lin-57/hbl-1 controls developmental time and is regulated by microRNAs. Dev Cell 5, 625-637 (2003).

14. Lin S.Y. et al. The $\mathrm{C}$ elegans hunchback homolog, hbl-1, controls temporal patterning and is a probable microRNA target. Dev Cell 4, 639-650 (2003).

15. Thompson-Peer K., Bai J., Hu Z. \& Kaplan J.M. HBL-1 patterns synaptic remodeling in C. elegans. Neuron 73, 453-465 (2012).

16. Grosskortenhaus R., Pearson B.J., Marusich A. \& Doe C.Q. Regulation of temporal identity transitions in Drosophila neuroblasts. Dev.Cell 8, 193-202 (2005).

17. Cho P.F. et al. Cap-dependent translational inhibition establishes two opposing morphogen gradients in Drosophila embryos. Curr Biol 16, 2035-2041 (2006). 
18. Dinkova T.D., Keiper B.D., Korneeva N.L., Aamot E.J. \& Rhoads R.E.Translation of a small subset of Caenorhabditis elegans mRNAs is dependent on a specific eukaryotic translation initiation factor 4E isoform. Mol Cell Biol 25, 100-113 (2005).

19. Dallman J.E., Allopenna J., Bassett A., Travers A. \& Mandel G. Conserved Role But Different Partners for the Transcriptional Corepressor CoREST in Fly and Mammalian Nervous System Formation. J. Neurosci. 24, 7186-7193 (2004).

20. Mandel G. et al. Repressor element 1 silencing transcription factor (REST) controls radial migration and temporal neuronal specification during neocortical development. Proc. Natl. Acad. Sci. USA 108, 16789-16794 (2011).

21. Ballas N., Grunseich C., Lu D.D., Speh J.C. \& Mandel G. REST and its corepressors mediate plasticity of neuronal gene chromatin throughout neurogenesis. Cell 121, 645-657 (2005).

22. Ballas N. \& Mandel G. The many faces of REST oversee epigenetic programming of neuronal genes. Curr. Opin. Neurobiol. 15, 500-506 (2005).

23. Jarriault S. \& Greenwald I. Suppressors of the egg-laying defective phenotype of sel-12 presenilin mutants implicate the CoREST corepressor complex in LIN-12/Notch signaling in C. elegans. Genes Dev 16, 2713-2728 (2002).

24. Bargmann C.I. Chemosensation in C. elegans. Wormbook, ed. C. elegans Research Community. Wormbook 10.1985/wormbook.1.123.1. http://www.wormbook.org (2006).

25. Altun Z.F. \& Hall D.H. Introduction WormAtlas, doi:10.3908/wormatlas.1 .1. (2009).

26. Yang M. et al. Structural basis for CoREST-dependent demethylation of nucleosomes by the human LSD1 histone demethylase. Mol. Cell 23, 377-387 (2006).

27. Zinovyeva A.Y., Graham S.M., Cloud V.J. \& Forrester W.C. The C.elegans histone deacetylase HDA-1 is required for cell migration and axon pathfinding. Dev. Biol. 289, 229242 (2006).

28. Van der Linden A.M., Nolan K.M. \& Sengupta P. KIN-29 SIK regulates chemoreceptor gene expression via an MEF2 transcription factor and a Class II HDAC. EMBO J. 26, 358370 (2007).

29. Renn M.C.P. \& Schumer M.E. Genetic assimilation and behavioural evolution: insights from genomic studies. Animal Behaviour 85, 1012-1022 (2013).

30. Forneris F., Binda C., Adamo A., Battaglioli E. \& Mattevi A. Structural basis of LSD1CoREST selectivity in histone H3 recognition. J Biol Chem 282, 20070-20074 (2007).

31. Lyons D.B. et al. An epigenetic trap stabilizes singular olfactory receptor expression. Cell 154, 325-336 (2013).

32. Wang Y. et al. LSD1 is a subunit of the NuRD complex and targets the metastasis programs in breast cancer. Cell 138, 660-672 (2009).

33. Denslow S.A. \& Wade P.A. The human Mi-2/NuRD complex and gene regulation. Oncogene 26, 5433-5438 (2007). 
34. Lan F., Nottke A.C. \& Shi Y. Mechanisms involved in the regulation of histone lysine demethylases. Curr Opin Cell Biol 20, 316-325 (2008).

35. Guan J.S. et al. HDAC2 negatively regulates memory formation and synaptic plasticity. Nature 459, 55-60 (2009).

36. Gräff J. et al. An epigenetic blockade of cognitive functions in the neurodegerating brain. Nature 29, 222-226 (2012).

37. West-Eberhard M.J. Developmental plasticity and the origin of species differences. Proc. Natl. Acad. Sci. USA 102, 6543-6549 (2005).

38. Kim J., Kerr J.Q. \& Min G.S. Molecular heterochrony in the early development in Drosophila. Proc. Natl. Acad. Sci. USA 97, 212-216 (2000).

39. Simola D.F., Francis C., Sniegowski P.D. \& Kim J. Heterochronic evolution reveals timing changes in budding yeast transcriptomes. Genome Biol. 11, R105 (2010).

40. Grant P.R. \& Grant B.R. Song of Darwin's finches diverge when a new species enters the community Proc. Natl. Acad. Sci. 107, 20156-20163 (2010).

41. Ginsburg S. \& Jablonka E. The evolution of associative learning: a factor in the Cambrian explosion J. Theor Biol. 266, 11-20 (2010). 


\section{Figures legends:}

\section{Figure 1}

\section{Implementation of chemo-attraction during physiological C.elegans development.}

BA 1/100 chemo-attraction of wild-type N2 worms was measured at 10, 26, 38, 56 and 60 hours post-hatch (blue curve). The mean migration indices (MMI) were calculated as indicated in Supplemental Material and Methods. Each chemotaxis assay included 20 worms and was repeated four times. The black curve depicts the wild-type growth curve of $C$. elegans (as described in WormAtlas, Altun, Z. F. and Hall, D. H. (ed.s). 2002-2006).

\section{Figure 2}

\section{Implementation of chemo-attraction depends on HBL-1}

A. Implementation of BA-chemo-attraction during early life in C.elegans mutants. Chemotaxis assays were performed at age 10, 15 and 26 hours post-hatch in N2, in hbl-1 (ve18 and mg285), ife-4 (ok320) mutants and in ttx-3::hbl-1 transgenic. Mean migration indices were calculated for each chemotaxis assay and compared to N2. Each chemotaxis assay included 20 worms and was repeated four times. ${ }^{* *}$ p-value $<0.01$.

\section{B. Implementation of chemo-attraction throughout development in C.elegans mutants.}

BA 1/100 chemo-attraction of wild-type N2, hbl-1, ife-4, and ttx-3::hbl-1 mutants was measured at 10, 15, 26, 56, 60 and 84 hours post-hatch. Mean migration indices were calculated for each chemotaxis assay and compared to N2. Each chemotaxis assay included 20 worms and was repeated four times.

\section{Figure 3}

\section{Olfactory imprinting is HBL-1 dependent.}

Wild-type N2 and hbl-1, ife-4 and ttx-3::hbl-1 mutants were exposed to BA 1/300 for 12 hours post-hatch in order to generate an olfactory imprinting. Chemotaxis assays of these odor-exposed and their non-exposed counterpart were then performed at adulthood. Mean imprinting indices to BA 1/300 were calculated by subtracting the MMI of non-exposed mutants from the MMI of odor-exposed mutants (detailed description in Material and Methods). Each chemotaxis assay included 20 worms and was repeated four times. ${ }^{* *}$ p-value $<0.01$.

\section{Figure 4}

Precocious implementation of chemo-attraction is negatively correlated to chemoattraction expression.

\section{A. Implementation of chemotaxis response to BA}

Mutant larvae aged of 26 hours post-hatch were assayed for chemo-attraction to BA 1/100. Mean migration indices were then calculated for each chemotaxis assay as described in Supplemental Material and Methods. Each chemotaxis assay included 20 worms and was repeated four times.

\section{B. Acquisition of olfactory imprinting}

Worms were exposed to BA $1 / 300$ for 12 hours post-hatch in order to generate an olfactory imprinting. Chemotaxis assays of these odor-exposed and their non-exposed counterpart were 
then performed during adulthood. Mean imprinting indices to BA 1/300 were calculated by subtracting the MMI of non-exposed mutants from the MMI of odor-exposed mutants (detailed description in Material and Methods). Each chemotaxis assay included 20 worms and was repeated four times.

\section{Figure 5}

\section{The rate of chemo-attraction behaviour reshaping is determined by the multigenerational accumulation of chemosensory experience.}

A. Worm mutants were exposed to BA 1/300 for 60 hours post-hatch in order to generate an olfactory imprinting. This odor-exposure was reiterated for several consecutive generations. To assess stable reshaping of the BA-chemoattraction, the F1 to F5 progeny issued from every BA-exposed generation (and from unexposed as control) were grown without BA exposure for 5 generations. Mean migration indices of the unexposed progeny - from F1 up to F5 - were determined and compared to the mean migration indices of naïve animals of the same genotype, as described. Each chemotaxis assay included 20 worms and was repeated four times.

B. Consecutive generations of ife-4 (ok320) worms were exposed (or unexposed for naïve controls) to BA1/300 during 60, 36 or 12 hours post-hatch. To assess stable reshaping of the BA-chemoattraction, the F1 to F5 progeny issued from the three imprinting intervals was assessed. Each chemotaxis assay included 20 worms and was repeated four times.

\section{Figure 6}

\section{A putative causal chain linking the timing of neuronal plasticity to the stable behaviour adaptation rate.}




\begin{tabular}{|c|c|c|c|c|c|c|}
\hline \multirow{3}{*}{$\begin{array}{l}\text { Worm } \\
\text { strain }\end{array}$} & \multicolumn{5}{|c|}{ BA 1/300 exposure time (post-hatch hours) } & \multirow{3}{*}{$\begin{array}{l}\text { Critical } \\
\text { period of } \\
\text { imprinting }\end{array}$} \\
\hline & Unexposed & 0 to $12 h$ & 0 to 60h & 12h to $36 \mathrm{~h}$ & $36 \mathrm{~h}$ to $60 \mathrm{~h}$ & \\
\hline & \multicolumn{5}{|c|}{ Mean migration index (+/- s.e.) } & \\
\hline $\begin{array}{l}\text { ife-4(ok320) } \\
n=5\end{array}$ & $1.7 \pm 0.3$ & $2.6 \pm 0.2$ & $3.3 \pm 0.25$ & $2.9 \pm 0.25$ & $3.4 \pm 0.3$ & $\begin{array}{l}>60 h \\
\text { post-hatch }\end{array}$ \\
\hline $\begin{array}{l}t t x-3:: h b l-1 \\
n=4\end{array}$ & $1.6 \pm 0.25$ & $2.8 \pm 0.28$ & nd & nd & $3.2 \pm 0.25$ & $\begin{array}{l}>60 h \\
\text { post-hatch }\end{array}$ \\
\hline $\begin{array}{l}\text { hda-2(ok1479) } \\
n=5\end{array}$ & $1.6 \pm 0.3$ & $3.2 \pm 0.3$ & $3.5 \pm 0.3$ & $3.3 \pm 0.25$ & $1.4 \pm 0.3$ & $\begin{array}{l}\text { 36h } \\
\text { post-hatch }\end{array}$ \\
\hline $\begin{array}{l}\text { N2 wild-type } \\
n=10\end{array}$ & $1.65 \pm 0.3$ & $3.6 \pm 0.25$ & $3.4 \pm 0.3$ & $1.8 \pm 0.2$ & $1.55 \pm 0.2$ & $\begin{array}{l}\text { 12h } \\
\text { post-hatch }\end{array}$ \\
\hline $\begin{array}{l}\text { hda-4(ok518) } \\
n=7\end{array}$ & $1.8 \pm 0.3$ & $3.4 \pm 0.3$ & $3.5 \pm 0.35$ & $2.1 \pm 0.3$ & $2.0 \pm 0.2$ & $\begin{array}{l}\text { 12h } \\
\text { post-hatch }\end{array}$ \\
\hline $\begin{array}{l}\text { hda-3(ok1991) } \\
n=7\end{array}$ & $1.55 \pm 0.25$ & $-0.4 \pm 0.2$ & $-0.3 \pm 0.18$ & $1.4 \pm 0.3$ & $1.7 \pm 0.2$ & $\begin{array}{l}\text { 12h } \\
\text { Negative }\end{array}$ \\
\hline
\end{tabular}

Table 1 Mutations that affect the timing of olfactory imprinting critical periods.

Worms of the indicated genotypes were either exposed or not exposed to BA 1/300 during the indicated periods of post-hatch development in order to generate an imprint. Each chemotaxis assay included 20 worms and the mean migration indices each are indicated (n=number of repeats). MMI for odor-exposed and for naïve unexposed worms were compared using the Student $t$ unpaired data with unequal variance test. All italic bold numbers indicate that pvalue $<0.01$. 


\begin{tabular}{|c|c|c|c|c|}
\hline Worm strain & \multicolumn{3}{|c|}{$\begin{array}{l}\text { BA 1/300 responses of the fifth unexposed generation } \\
\text { after } \mathrm{N} \text { generations (Ngen) were odor-exposed }(0-60 \mathrm{~h})\end{array}$} & $\begin{array}{l}\text { Generation from } \\
\text { which imprinting } \\
\text { switch to stable }\end{array}$ \\
\hline ife-4(ok320) & $\begin{array}{l}\text { Ngen=1 } \\
3.6 \pm 0.2\end{array}$ & $\begin{array}{l}\text { Ngen=4 } \\
3.4 \pm 0.2\end{array}$ & $\begin{array}{l}\text { Ngen=5 } \\
\mathbf{3 . 6} \pm \mathbf{0 . 3}\end{array}$ & FIRST \\
\hline $\begin{array}{l}\text { Transgenics } \\
\text { ttx-3 ::hbl-1 }\end{array}$ & $\begin{array}{l}\text { Ngen }=1 \\
3.2 \pm 0.3\end{array}$ & $\begin{array}{l}\text { Ngen=4 } \\
3.0 \pm 0.2\end{array}$ & $\begin{array}{l}\text { Ngen=5 } \\
\mathbf{3 . 8} \pm \mathbf{0 . 3}\end{array}$ & FIRST \\
\hline hda-2(ok1479) & $\begin{array}{l}\text { Ngen }=1 \\
1.5 \pm 0.3\end{array}$ & $\begin{array}{l}\text { Ngen=4 } \\
3.0 \pm 0.3\end{array}$ & $\begin{array}{l}\text { Ngen=5 } \\
3.2 \pm 0.3\end{array}$ & FOURTH \\
\hline N2 wild-type & $\begin{array}{l}\text { Ngen }=1 \\
1.5 \pm 0.3\end{array}$ & $\begin{array}{l}\text { Ngen }=4 \\
1.8 \pm 0.25\end{array}$ & $\begin{array}{l}\text { Ngen }=5 \\
3.2 \pm 0.3\end{array}$ & FIFTH \\
\hline hda-4(ok518) & $\begin{array}{l}\text { Ngen }=1 \\
1.7 \pm 0.3\end{array}$ & $\begin{array}{c}\text { Ngen }=4 \\
1.8 \pm 0.3\end{array}$ & $\begin{array}{l}\text { Ngen=5 } \\
3.3 \pm 0.35\end{array}$ & FIFTH \\
\hline hda-3(ok1991) & $\begin{array}{l}\text { Ngen }=1 \\
-0.5 \pm 0.2\end{array}$ & $\begin{array}{l}\text { Ngen }=4 \\
-0.25 \pm 0.3\end{array}$ & $\begin{array}{l}\text { Ngen=5 } \\
-\mathbf{0 . 4 \pm 0 . 2}\end{array}$ & FIRST \\
\hline
\end{tabular}

Table 2 Prolonged imprinting periods accelerate behavioural reshaping in worm populations.

Worm mutants were exposed to BA $1 / 300$ for 60 hours post-hatch in order to generate an olfactory imprinting. This odor-exposure was reiterated for 1,4 or 5 consecutive generations (Ngen=1, Ngen=4 or Ngen=5). To assess stable reshaping of the BA-chemoattraction, the F1 to F5 progeny issued from each BA-exposed generation (and from unexposed as control) were grown without BA exposure for 5 generations. Mean migration indices of the unexposed progeny - from F1 up to F5 - were determined and compared to the mean migration indices of naïve animals of the same genotype, as described. Each chemotaxis assay included 20 worms and was repeated four times. All italic bold numbers indicate that p-value $<0.01$. 
Figure 1

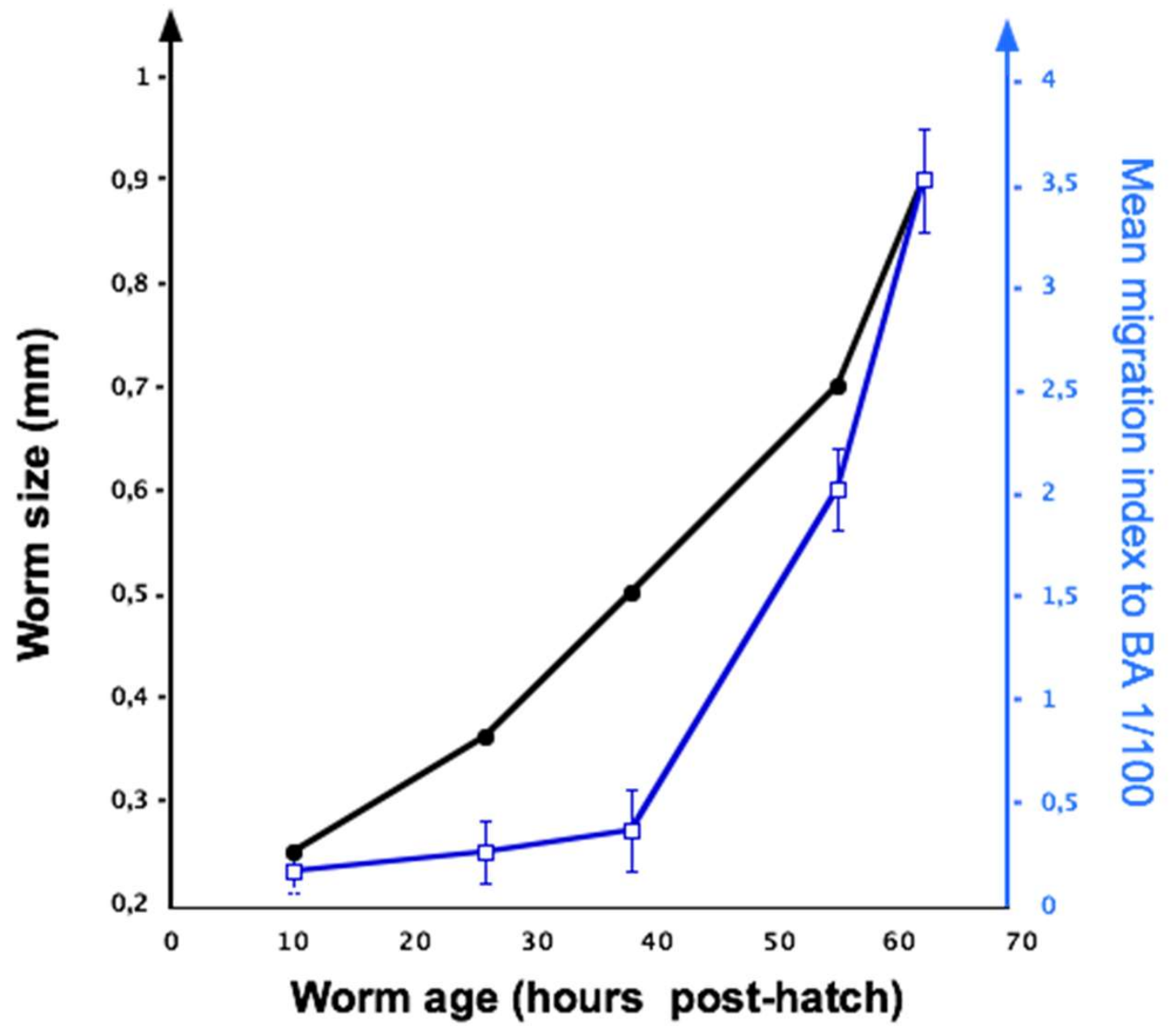


Figure 2
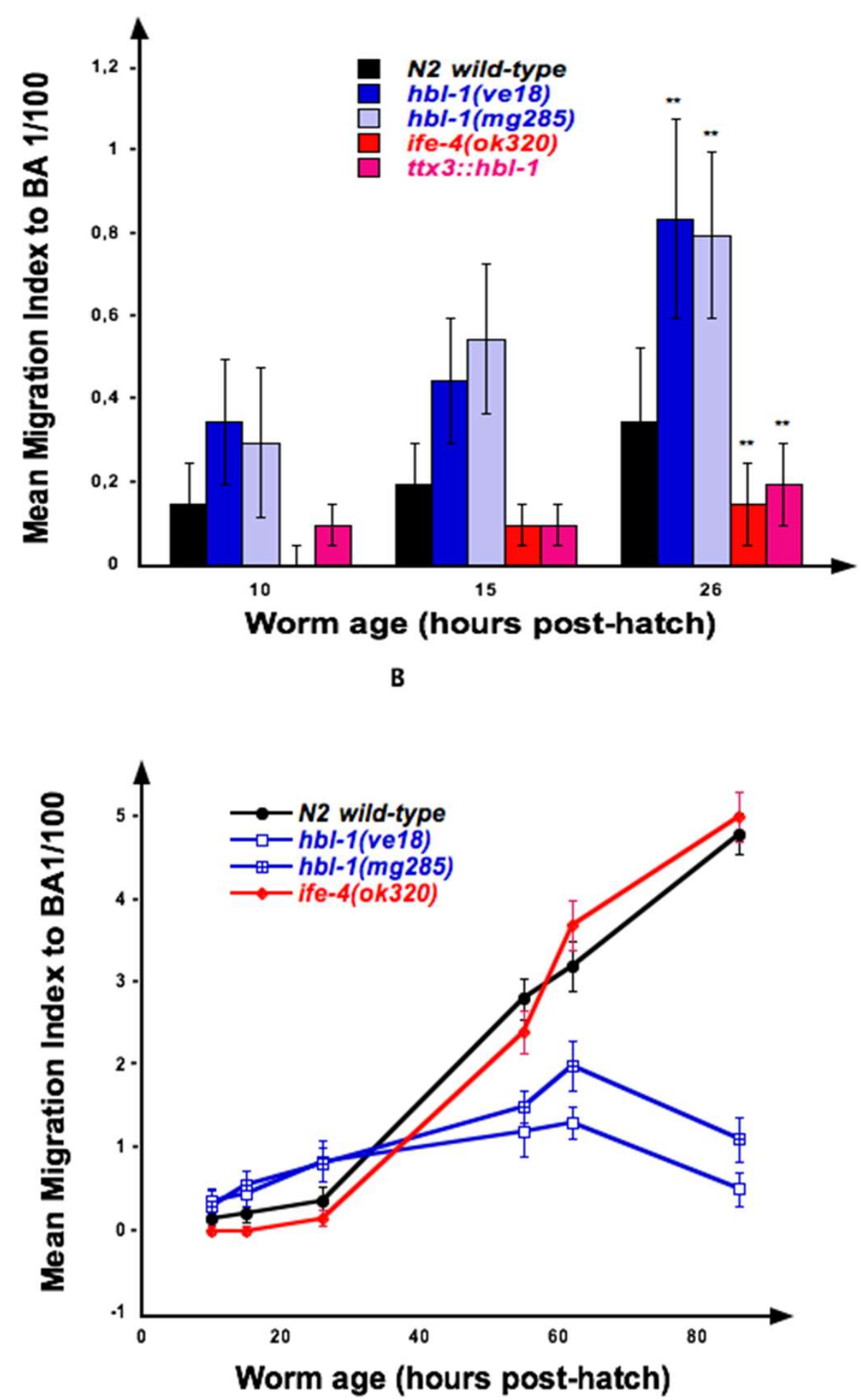
Figure 3

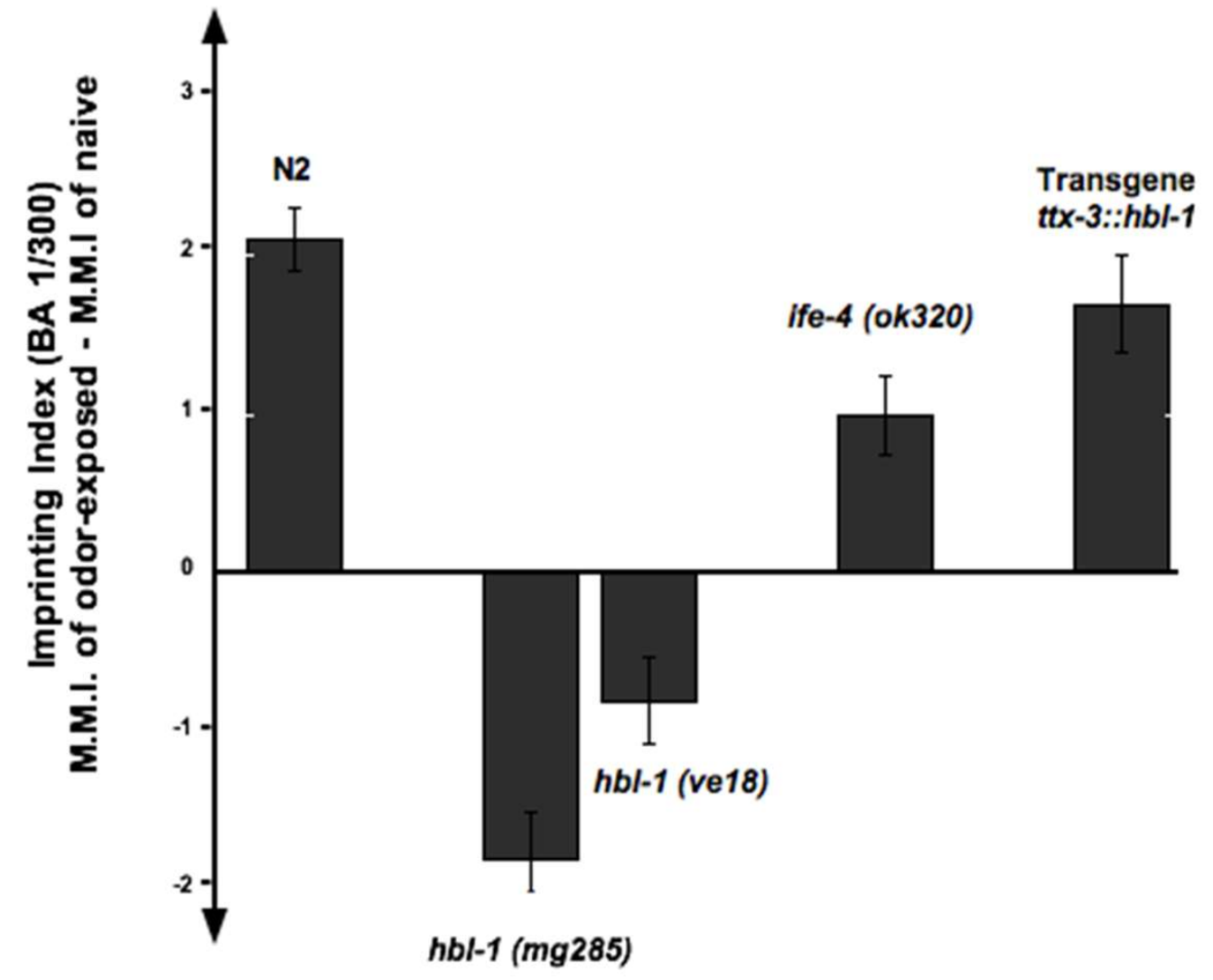


Figure 4
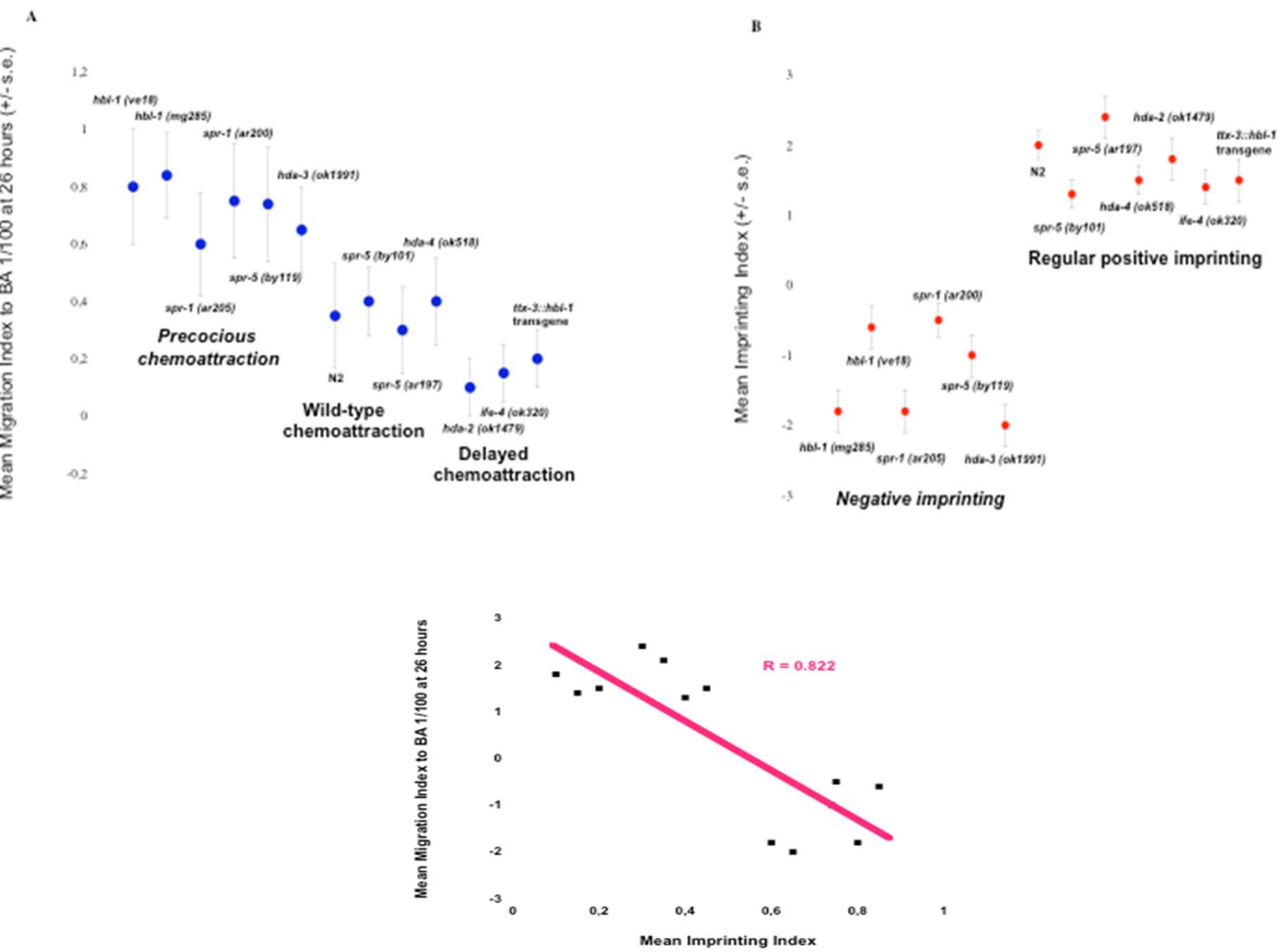
Figure 5

A Multigenerational BA exposure

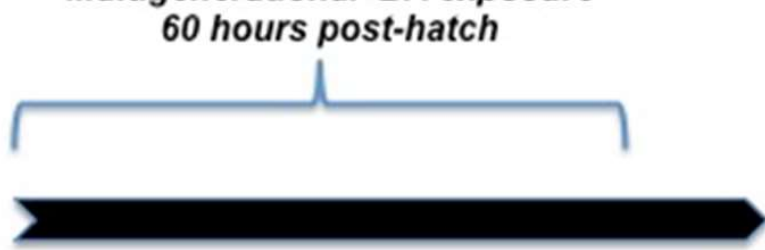

$>$

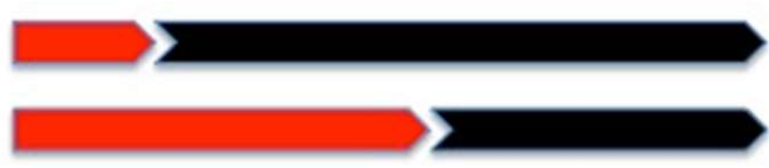

E

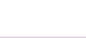

\section{Worm strains}

Critical period

BA imprinting

hbl-1 (mg285), hbl-1 (ve18)

spr-1 (ar205), spr-1 (ar200)

spr-5 (by119), hda-3 (ok1991)

N2 wild-type, hda-4 (ok518) spr-5 (ar197), spr-5 (ar197)

hda-2 (ok1479)

ife-4 (ok320)

ttx-3::hbl-1 transgene
Negative imprinting

12 hours

36 hours

60 hours
Stable inheritance after

\section{5 generations \\ 4 generations \\ 1 generation}

B Multigenerational BA exposure

$$
60 \text { hours post-hatch }
$$

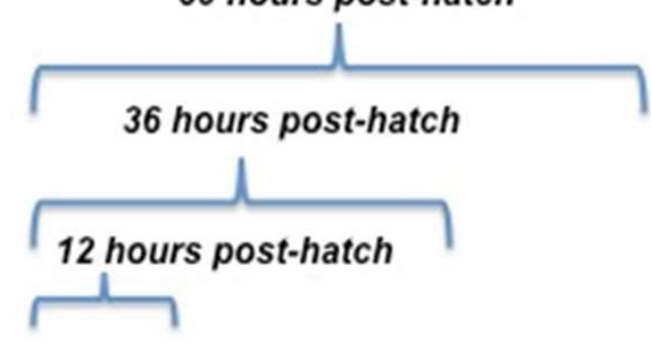

ife-4 (0k320)

60 hours

5 generations

Ife-4 (ok320)

60 hours

4 generations

Ife-4 (ok320)

60 hours

1 generation 
Figure 6

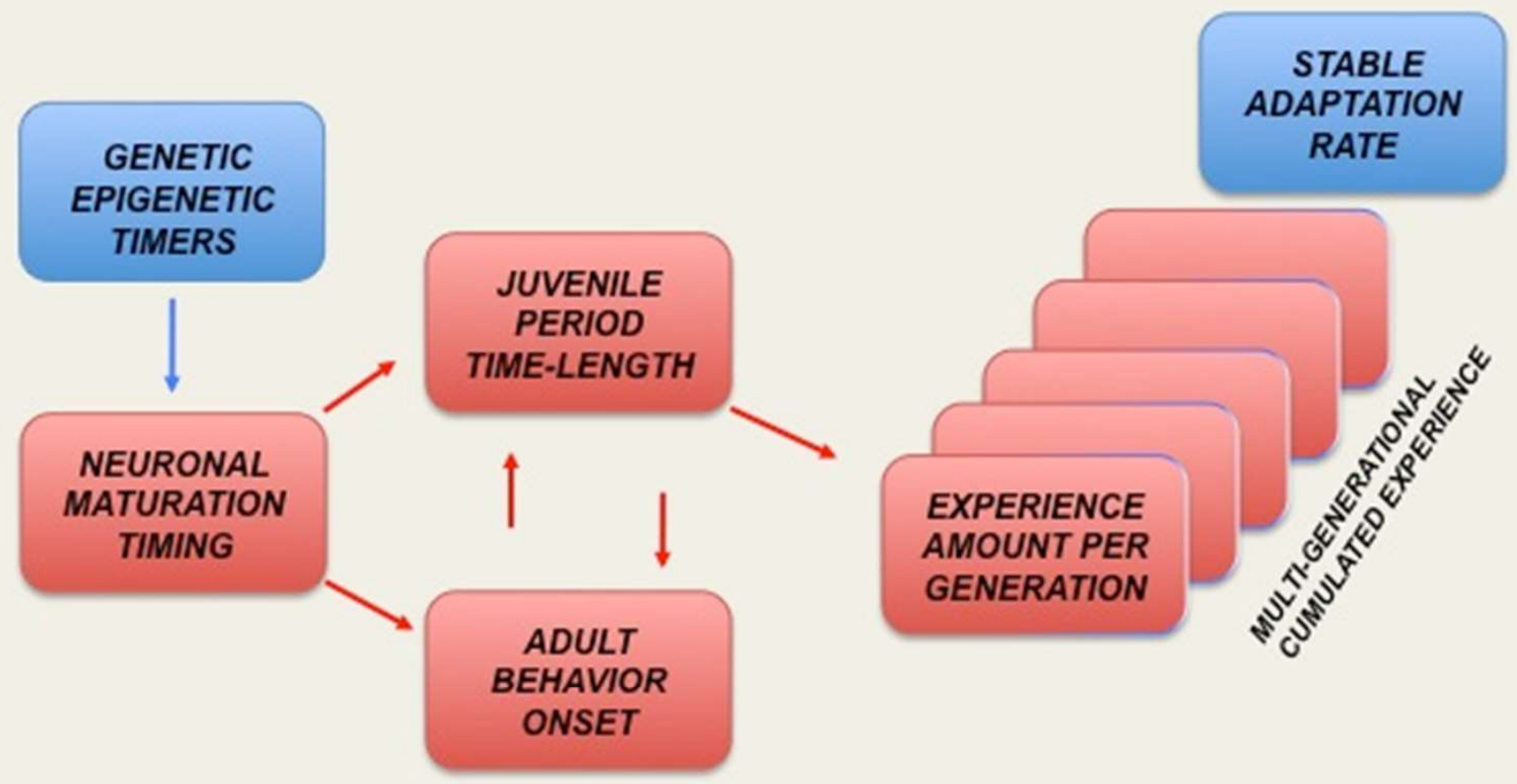

A putative causal chain linking timing of neuronal plasticity and stable behavior adaptation rate 